\title{
Going beyond 1000000 resolved points in a Brillouin distributed fiber sensor: theoretical analysis and experimental demonstration
}

\author{
Andrey Denisov, Marcelo A Soto and Luc Thévenaz
}

Distributed fiber sensing possesses the unique ability to measure the distributed profile of an environmental quantity along many tens of kilometers with spatial resolutions in the meter or even centimeter scale. This feature enables distributed sensors to provide a large number of resolved points using a single optical fiber. However, in current systems, this number has remained constrained to a few hundreds of thousands due to the finite signal-to-noise ratio (SNR) of the measurements, which imposes significant challenges in the development of more performing sensors. Here, we propose and experimentally demonstrate an ultimately optimized distributed fiber sensor capable of resolving 2100000 independent points, which corresponds to a one-orderof-magnitude improvement compared to the state-of-the-art. Using a Brillouin distributed fiber sensor based on phase-modulation correlation-domain analysis combined with temporal gating of the pump and time-domain acquisition, a spatial resolution of $8.3 \mathrm{~mm}$ is demonstrated over a distance of $17.5 \mathrm{~km}$. The sensor design addresses the most relevant factors impacting the SNR and the performance of medium-to-long range sensors as well as of sub-meter spatial resolution schemes. This step record in the number of resolved points could be reached due to two theoretical models proposed and experimentally validated in this study: one model describes the spatial resolution of the system and its relation with the sampling interval, and the other describes the amplitude response of the sensor, providing an accurate estimation of the SNR of the measurements.

Light: Science \& Applications (2016) 5, e16074; doi:10.1038/Isa.2016.74; published online 6 May 2016

Keywords: distributed fiber sensors; optical fibers; stimulated Brillouin scattering

\section{INTRODUCTION}

After more than two decades of intense research and development, distributed optical fiber sensing has become a mature technology, showing clear advantages over its traditional counterpart based on discrete sensing elements. A single distributed system is able to continuously interrogate a large number of independent points (given by the quotient between the sensing range and the spatial resolution) along an optical fiber. This feature opens unique opportunities to monitor, for example, large civil structures or very long pipelines in the oil and gas industry, where a large number of resolved points are typically required.

One of the techniques that offers the highest performance, and thus provides the largest number of resolved points, is Brillouin optical time-domain analysis (BOTDA) $)^{1}$. Using an optimized conventional BOTDA scheme, the sensing range can reach $50 \mathrm{~km}$, and with the help of advanced techniques, such as Raman amplification ${ }^{2-4}$, optical pulse coding $^{5-11}$ or a combination of them ${ }^{6,12,13}$, this range can be extended beyond $100 \mathrm{~km}$. However, the spatial resolution of a BOTDA system is ultimately limited to $1 \mathrm{~m}$ by the acoustic-wave response time ${ }^{14}$; therefore, dedicated techniques must be used to reach the sub-meter resolution. Methods such as correlation domain ${ }^{15-17}$, acoustic pre-activation ${ }^{18-20}$, or differential pulses ${ }^{21,22}$, among others, have allowed the spatial resolution to be improved to the order of centimeters or millimeters, but only along restricted fiber lengths (less than $5 \mathrm{~km}$ long) ${ }^{21-23}$.

Due to the difficulties faced in combining sub-meter spatial resolutions with large sensing ranges, the maximum number of resolved points along a single fiber has basically remained limited to $\sim 100000$ points $^{24}$, e.g., $1 \mathrm{~m}$ over $120 \mathrm{~km}^{6}$ or $2 \mathrm{~cm}$ over $2 \mathrm{~km}^{23}$. Currently, intense research activities are underway to further enhance the sensor performance and increase the number of points that a single system can resolve. However, increasing this number beyond the state-of-the-art turns out to be extremely challenging due to the weak Brillouin interaction and low signal-to-noise ratio (SNR) resulting from a fine spatial resolution or very long sensing ranges ${ }^{24}$. Recently, 240000 resolved points have been demonstrated over a 60-km-long fiber using a combination of Raman amplification, pulse coding, and differential pulse-width pair technique ${ }^{25}$, and 300000 points have been resolved over $3.3 \mathrm{~km}$ using a correlation-domain approach ${ }^{26}$. A significant increase in the number of resolved points, for example, by one order of magnitude, requires a smart combination of schemes for sub-meter resolutions and techniques to address the 
limitations existing at long sensing ranges (i.e., to compensate the high fiber attenuation and to mitigate the impact of nonlinearities).

The phase-modulated correlation-domain method has been proven to be a promising technique to combine long sensing ranges with fine spatial resolution ${ }^{17,27}$. This method is based on the phase modulation of the interacting optical waves-pump and probe-using a pseudorandom bit sequence (PRBS), which confines the Brillouin interaction to a set of discrete points referred to as correlation peaks. One of the main factors limiting the performance of the technique is the residual random Brillouin interaction occurring outside of the correlation peaks. This interaction actually generates noise that scales linearly with the fiber length, ultimately limiting the number of resolved points to $\sim 100000$. Recently, it has been demonstrated that this noise can be significantly decreased by gating the phase-modulated pump wave with square pulses and using time-domain detection of the signal wave 26,28 , which also enables simultaneous measurement of the Brillouin gain (or loss) at multiple correlation peaks along the fiber. In a recent work, London et al. ${ }^{29}$ reported 110000 resolved points along a $2.2-\mathrm{km}$ fiber, also demonstrating a considerable reduction in the measurement time.

This study presents a thorough theoretical analysis and an optimization procedure of the critical parameters defining the performance of Brillouin sensors based on a phase-modulated correlation-domain technique combined with time-domain acquisition. In particular, the classical definition of the spatial resolution and its relation with the sampling interval are revisited for this type of systems. Based on this analysis and optimizing previous implementations ${ }^{26,27}$, distributed sensing along a $17.5-\mathrm{km}$ fiber is experimentally verified with a spatial resolution of $8.3 \mathrm{~mm}$. This distributed sensing demonstrates the capability of the system to resolve 2100000 distinct points along a single optical fiber, which corresponds to a significant boost in the performance of Brillouin distributed fiber sensors and constitutes a significant breakthrough in the field.

\section{MATERIALS AND METHODS}

\section{Combining time and correlation domain analysis}

Phase-correlation distributed Brillouin sensors use phase modulation over the interacting waves to localize the Brillouin interaction at welldefined positions along the fiber ${ }^{17}$. A single binary sequence is used to modulate the phases of both pump and probe waves. The sequence can be either a PRBS ${ }^{17,26,27}$ or any other suitable code sequence, such as the Golomb code ${ }^{28}$. In this work, the phases of both pump and probe waves are modulated with a PRBS, applying a phase shift of 0 or $\pi$; however, note that the descriptions and analysis presented in the current study also hold for any other suitable code sequence.

Stimulated Brillouin scattering is based on the interference between counter-propagating pump and probe waves; therefore, it depends on the local phase difference between the complex amplitudes of these waves. For the above-mentioned phase modulation, this local phase difference takes the values of 0 or $\pi$. Because the same periodic pattern is used to modulate the phases of the pump and the probe, equal counter-propagating phases will meet at very specific short sections of fiber (correlation peaks) separated by a distance given by the PRBS period ${ }^{17}$. At other fiber locations, the phases of the two waves are absolutely temporally uncorrelated due to the random nature of the sequence, and the difference between them switches randomly between 0 and $\pi$ so that the time-averaged value of the interference amplitude is zero.

Although the bit duration is two orders of magnitude shorter than the acoustic phonon lifetime, the constant phase difference between the pump and the signal at the correlation peaks allows the
Brillouin-induced acoustic wave to increase to its steady-state level, forming a sustained dynamic Bragg grating ${ }^{17,30}$. The size of this grating is given by the bit duration, e.g., $1 \mathrm{~cm}$ full-width at half-maximum (FWHM) for a bit length of 100 ps (see Section S1 of the Supplementary material for details). In this manner, the Brillouin interaction is probed over only this small fiber section, avoiding the detrimental spectral broadening of the Brillouin gain affecting time-domain measurements. To perform a measurement along the whole sensing fiber, the correlation peaks must be repositioned to scan the fiber point-by-point ${ }^{17}$.

As mentioned before, outside the correlation peaks, the interaction between the pump and the signal randomly changes sign due to the uncorrelated phase difference between these waves, generating weak random gratings ${ }^{17,30}$. Pump-probe interaction at these random gratings generates noise that is proportional to the fiber length for continuous interacting waves and can severely affect the performance of the system when long sensing fibers are used. This problem is solved by a temporal gating of the pump combined with a timedomain measurement of the probe ${ }^{26,28}$. This approach leads to a significantly lower noise originating from random gratings, making larger sensing ranges possible and enabling the simultaneous measurement of multiple correlation points along the fiber.

\section{Limiting factors}

The performance of a distributed Brillouin fiber sensor, given essentially by the uncertainty on the determination of the Brillouin frequency shift (for a given spatial resolution and fiber length), is ultimately determined by the SNR of the measurements ${ }^{31}$. Thus, to optimize the system to enable the best possible performance, it is of utmost importance to determine the factors that limit the SNR in a Brillouin sensor combining phase-modulated correlation-domain and time-domain measurements. For a fixed spatial resolution, the useful signal amplitude is given by the pump and probe powers (both limited by the threshold of nonlinear effects ${ }^{32,33}$ ) and fiber losses ${ }^{31}$. Usually, sensors with sub-meter resolution show a limited sensing range of several hundred meters or a few kilometers so that limitations imposed by the fiber attenuation can be generally neglected, whereas the pump power can be as high as several watts with no onset of nonlinear effects. However, as the length of the fiber increases (e.g., going beyond $10 \mathrm{~km}$ ), the fiber losses and nonlinear effects can become significant. In such a situation, the main limiting nonlinear effect is modulation instability, which imposes a maximum pump power of $100 \mathrm{~mW}$ for fibers longer than $20 \mathrm{~km}^{32,33}$. Additional limitation comes from the fact that the spectrum of the phasemodulated pump consists of multiple lines separated by a few $\mathrm{MHz}$; therefore, it is very prone to four-wave mixing.

Furthermore, if the range of a phase-correlation Brillouin sensor is extended while maintaining centimeter or millimeter resolution, an SNR optimization not only requires addressing the factors limiting the sensor response at long distances but also should reduce the different sources of noise affecting the measurements. In addition to the common shot and thermal noises present in any optical system ${ }^{24}$, there are other sources of noise specific to the phase-correlation technique. As discussed before, outside the correlation peaks, the acoustic wave amplitude follows a random distribution, leading to a noise-like interaction between pump and probe ${ }^{17}$. In addition, in real systems, there always exists a bandwidth limitation in the phase modulation, leading to non-instantaneous phase transitions, which gives rise to stable weak gratings being generated along the fiber at each bit transition edge that becomes more pronounced for higher spatial resolutions as the PRBS bit rate approaches the system's 
bandwidth limitation (see Section S2 of the Supplementary material for details). Because the phases of pump and probe are not correlated at these positions, the pump-signal energy transfer is also random, leading to additional noise. Although the existence of this second source of noise has not yet been reported in the literature, it can have a significant detrimental impact on the performance of the sensor, especially when aiming at very high spatial resolutions (e.g., a few $\mathrm{mm}$ or sub-mm). Considering that these two sources of noise scale with the duration of the pump pulse, a suitable optimization of the pulse length is required to maximize the SNR of the measurements.

\section{Increasing the number of points}

Because both directions to improve the sensor performance (i.e., increasing the sensing range and refining the spatial resolution) can considerably reduce the $\mathrm{SNR}^{24}$, the measurement time scales highly nonlinearly with the number of points in the system. For example, a twofold improvement in the spatial resolution decreases the SNR by a factor of 2, which must be compensated by raising the number of averages by a factor of 4 . Furthermore, it should be considered that the number of simultaneously measured points along the fiber is given by the separation between correlation peaks, limited by the pump pulse duration, which remains constant. Thus, the measurement time is increased by an additional factor of 2 resulting from the twofold increase in the number of points to be scanned. As a consequence, the overall measurement time increases by a factor of 8 , representing a cubic dependence of the acquisition time on the spatial resolution. If the sensing range is increased, then the impact on the measurement time is not as simple to estimate because the thresholds for nonlinear effects (depending on the effective nonlinear length of the fiber), fiber losses and the additional measurement time coming from the longer time of flight must be taken into account. However, overall, increasing the number of points by extending the sensing distance leads to a considerably longer measurement time than in the case of improving the spatial resolution.

Spatial resolution and impact of the sampling interval

By definition, the spatial resolution of the system is given by the shortest detectable scaled change in the Brillouin frequency. Consider a uniform fiber with a certain Brillouin frequency $v_{0}$ and a distinct uniform section of length $d$ with a different, well-separated Brillouin frequency $v_{\mathrm{h}}$, referred hereafter as hotspot. (The well-separated frequency is selected here for clarity; however, the presented model and description are also valid for small frequency differences.) In this configuration, the gain at a frequency offset $v_{0}$ sharply drops to zero in the hotspot position, whereas the gain at the frequency $v_{\mathrm{h}}$ goes from zero to its maximum value. However, the measured gain values behave differently due to the finite spatial resolution of the system.

The gain response of the sensor is given locally by the overlap integral of the acoustic wave amplitude and a rectangular function given by the hotspot size. For the case of a perfect square phase modulation, the acoustic wave amplitude within the correlation peak can be easily found to have a triangular shape with a FWHM given by the PRBS bit duration, e.g., a FWHM of $14 \mathrm{~mm}$ for a bit duration of 140 ps. (Details on the correlation peak shape can be found in Section S1 of the Supplementary material, including the more realistic case of non-instantaneous transition between two phase states, i.e., the limited bandwidth of the phase modulation.) Figure 1 shows the simulated gain trace along the fiber for three distinct hotspot sizes, for frequencies $v_{0}$ and $v_{\mathrm{h}}$. The shaded area of the correlation peak represents its overlap with the hotspot. Here, a hotspot is considered detectable if the measured gain at $v_{\mathrm{h}}$ dominates over a given fiber section and thus exceeds the gain at $v_{0}$. Based on the above consideration, the three cases in Figure 1 represent the conditions of (a) undetectable, (b) barely detectable, and (c) well-detected hotspot.

It is important to remember that in a real system both the spatial resolution and the sampling interval are finite. To reliably detect a hotspot, the sampling interval cannot be longer than the fiber segment, where the measured gain at $v_{\mathrm{h}}$ exceeds the gain at $v_{0}$, which is equivalent to the gain at $v_{\mathrm{h}}$ exceeding 0.5 times its maximum possible value (Figure 1c). If the sampling interval is longer than the hotspot section $s$, then the hotspot will not be reliably detected (the sampling can simply skip the hotspot region). Under this consideration, in the limit case presented in Figure 1b, the hotspot is only theoretically detectable with a continuous sampling.

The value of the sampling interval required to achieve a given spatial resolution can be found by calculating the sensor response; a detailed analysis of this method can be found in Section S2 of the Supplementary material. Figure 2 shows the maximum sampling interval required to secure a given spatial resolution for a bit duration of 140 ps (for perfect rectangular phase modulation and the case of bandwidth-limited phase modulation, with noninstantaneous transitions between the two phase states). It can be seen that the spatial resolution can reach $7.2 \mathrm{~mm}$, almost half of the correlation peak's FWHM. This is a direct consequence of the triangular spatial distribution of the acoustic wave, which gives a larger central ponderation. However, such a limiting case would require a continuous sampling. a

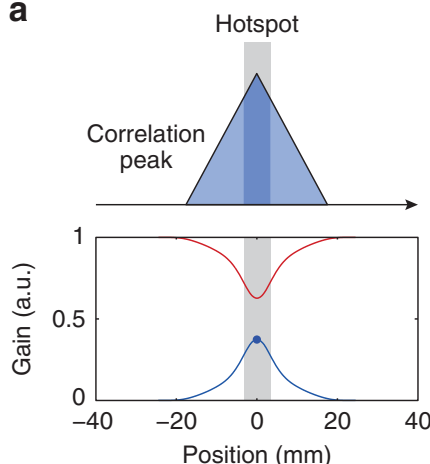

b

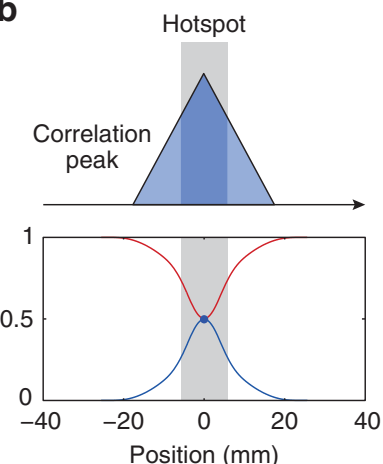

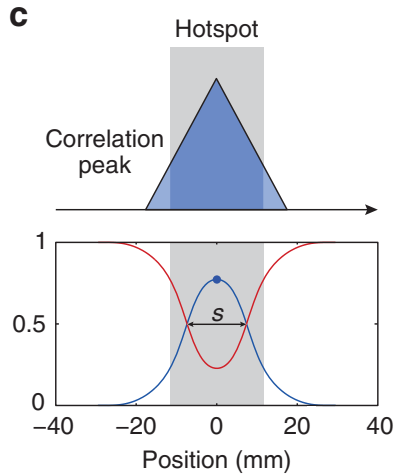

Figure 1 Simulated longitudinal Brillouin gain response for $v_{0}$ (red line) and $v_{h}$ (blue line), around hotspots of different lengths: (a) short undetected, (b) barely detected and (c) long well-detected hotspot. 


\section{RESULTS AND DISCUSSION}

\section{Experimental setup}

Aiming at demonstrating the ability to resolve more than 2000000 independent points along a single optical fiber, the experimental setup shown in Figure 3 was implemented. Monochromatic light from a $40-\mathrm{mW}$ distributed feedback laser diode with a linewidth of $1 \mathrm{MHz}$, operating at $1550.9 \mathrm{~nm}$, is guided through a phase modulator driven by a PRBS, which applies a phase shift of 0 or $\pi$ according to the sequence. Two PRBS lengths have been used in the experiment: $2^{15}-1$ (32 767 bits) and $2^{10}-1$ (1023 bits), which will be referred to hereafter as long and short PRBS, respectively. The bit duration is set to $140 \mathrm{ps,}$ similar to the value presented in the previous section; this way, Figure 2 can be straightforwardly used to determine the spatial resolution and the required sampling interval of the system. The phase-modulated light is then divided into two branches by an optical coupler. The lower branch in the figure is used to generate

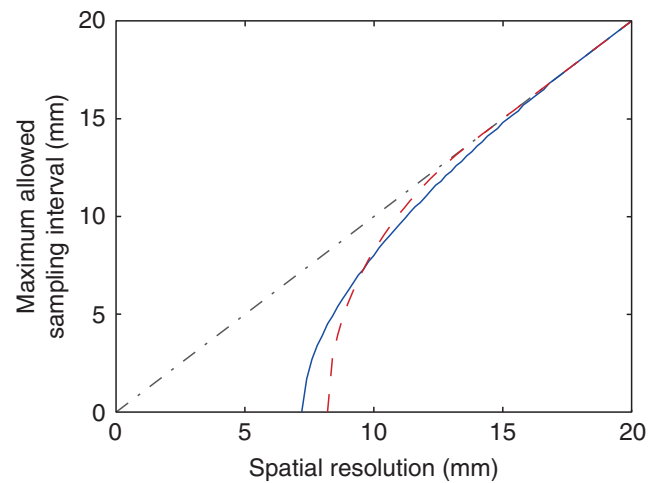

Figure 2 Maximum allowed sampling interval required to obtain a given spatial resolution when using a bit duration of $140 \mathrm{ps}$ for a noninstantaneous phase modulation (solid blue line) and a perfect square modulation (dashed red line). The dash-dotted black line represents the asymptotic case commonly used in the state-of-the-art, considering a sampling interval equal to the spatial resolution. pump pulses. First, the light is frequency shifted using a MachZehnder modulator driven by a microwave generator, and the longer-wavelength sideband is selected by tuning a 10-GHz-wide fiber Bragg grating. Next, pulses with 500 ps rise/fall time and high extinction ratio (more than $50 \mathrm{~dB}$ ) are generated by gating a semiconductor optical amplifier. The pulse width has been changed between 30 and $200 \mathrm{~ns}$ to characterize the SNR and measurement time of the system. An erbium-doped fiber amplifier boosts the peak pump power to $24 \mathrm{dBm}$ - a level optimized to maximize the sensor response and simultaneously avoid nonlinear effects in the fiber ${ }^{32,33}$.

The upper branch in Figure 3 is used to generate the probe wave at the nominal laser frequency. The phase-modulated probe passes through a $50-\mathrm{km}$-long delaying fiber and a polarization switch used to mitigate the polarization dependence of the Brillouin interaction. The long delay is required to allocate high-order correlation peaks inside the sensing fiber (a technique commonly used in correlationbased systems ${ }^{15,17}$ ) and can be implemented by any other method, for example, using two separate phase modulations for pump and probe and an electrically delayed modulation sequence. An erbium-doped fiber amplifier is used to compensate for the losses in the delaying fiber; the probe is then attenuated to $-10 \mathrm{dBm}$ and launched into a $17.5-\mathrm{km}$-long standard single-mode fiber. At the receiver, the signal is directed onto a $125-\mathrm{MHz}$ photo-detector followed by a $15-\mathrm{MHz}$ low-pass filter for noise reduction.

Note that the setup in Figure 3 corresponds to an optimized configuration compared to previous implementations ${ }^{26,27}$. In particular, the fiber Bragg grating, previously placed in the receiver side to filter out one of the probe sidebands, has been moved to the branch generating the pump, along with the double-sideband modulation. The spectral filtering of broadband modulated signals, as used in this method, introduces strong intensity noise (originating from phase to intensity conversion). Therefore, if this filter is placed in the probe propagation path, a huge intensity noise is directly introduced over the probe, with a significant detrimental impact on the measurements. In this case, the filter and the two-sideband modulation are located in the

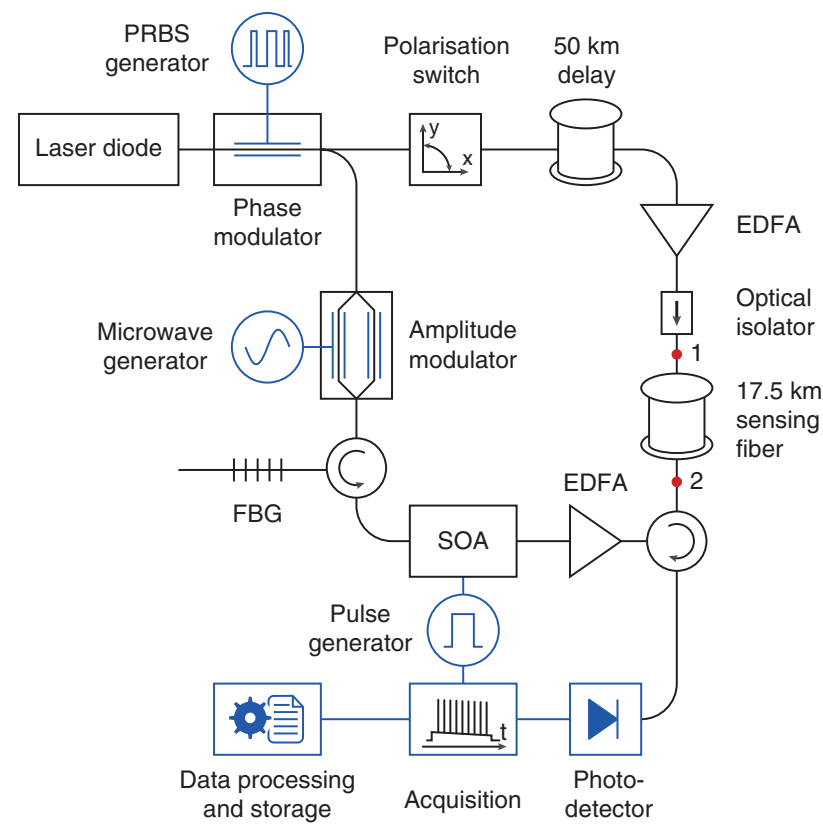

Figure 3 Experimental setup. Red dots show the two sensing fiber connectors used for measurements. Abbreviations: EDFA, erbium-doped fiber amplifier; FBG, fiber Bragg grating; SOA, semiconductor optical amplifier. 
pump branch, keeping the intensity noise restricted to the pulse duration. In addition, and more importantly, the low intensity-noise transfer from pump to probe in stimulated Brillouin scattering ${ }^{34,35}$ enables this noise to have a negligible impact on the measurements, which allows for more than a 10-fold improvement in the measurement speed (due to the lower number of averages) over previous schemes $^{26,27}$. The only effect that this new implementation could have is related to the Rayleigh scattering generated by the pump that reaches the photo-detector. However, this Rayleigh scattering is naturally spectrally flat; therefore, this component has no real impact on the spectral measurements of the Brillouin gain and can be easily eliminated by a simple data processing if needed.

\section{Time-domain traces and pump pulse optimization}

Using a sampling interval of $4.7 \mathrm{~mm}$, the sensing fiber is scanned with a spatial resolution of $8.3 \mathrm{~mm}$ (Figure 2). Figure 4 shows two timedomain traces acquired at the peak Brillouin gain frequency along the sensing fiber (averaged 512 times), for long (Figure 4a) and short PRBS (Figure 4b), using a 70-ns pump pulse. (This pulse length is chosen at this stage only for illustrative purposes; however, as it will be described later, this pulse length corresponds to the optimal length that maximizes the SNR and minimizes the measurement time.) Note that the correlation peak amplitudes vary slightly along the fiber because of the variations of the Brillouin frequency. For the long PRBS, correlation peaks are widely separated (every $460 \mathrm{~m}$ ) and a relatively strong background signal is observed. For the short PRBS, the separation between correlation peaks becomes much smaller $(14 \mathrm{~m})$ and almost no background signal is observed. The background signal actually originates from the multiple Brillouin cross-interactions existing between pump and probe spectral components (see the insets in Figure 4). For this reason, this cross-interaction and the resulting Brillouin gain highly depend on the spectral content of the two phasemodulated signals, which consist of multiple lines spectrally separated by the PRBS repetition rate: $215 \mathrm{kHz}$ and $6.8 \mathrm{MHz}$ for the long and short PRBS, respectively. Because the width of the Brillouin gain is $30 \mathrm{MHz}$, each spectral line of the pump wave can interact with more than 100 lines in the probe for the long PRBS case, giving rise to the background component observed in Figure 4a. For the short PRBS, the amount of cross-interaction is significantly reduced, leading to a negligible background component, as illustrated in Figure 4b.

One of the parameters of the system that must be optimized to achieve the best performance is the duration of the pump pulse. The pulse duration actually has multiple distinct effects on the measured traces. For pulses shorter than $\sim 30 \mathrm{~ns}$, the acoustic wave at each correlation peak does not have enough time to reach the steady-state condition, leading to a reduced sensor response; in addition, short

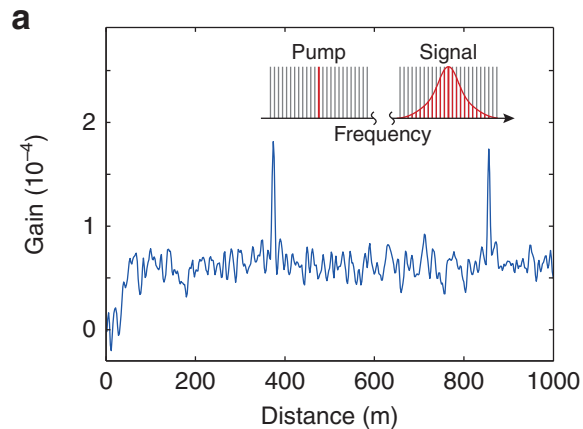

pulses also require large detection bandwidth, which increases the noise on the measurements. In contrast, longer pulses introduce more noise accumulated from randomly generated gratings outside the correlation peaks, weak permanent gratings coming from the non-instantaneous phase change and other spurious effects, thus leading to a significant decrease in SNR. Furthermore, the pump pulse duration defines the minimum separation between consecutive correlation peaks so that their responses do not overlap; therefore, an optimum pulse width has to be found to also minimize the measurement time. To determine the optimal pulse duration, the SNR of the measurements and the acquisition time have been evaluated for pump pulse widths ranging from 30 up to $200 \mathrm{~ns}$, for the long PRBS; the procedure was repeated for the short PRBS, but in this case, a maximum pulse duration of $130 \mathrm{~ns}$ was used to avoid significant overlapping of the correlation peaks. In this experiment, the electrical low-pass filter in the receiver was removed to avoid signal distortion for short pulse durations. However, to optimize the SNR, the measurement bandwidth was matched to the inverse of the pump pulse duration by means of digital filtering.

Figure $5 \mathrm{a}$ shows the SNR of the measurement at the average Brillouin frequency for both analyzed cases as a function of the pulse duration, as well as the theoretical SNR predicted using the measured noise (see Section S3 of the Supplementary material). The measurements are normalized to the SNR obtained with a pulse duration of $70 \mathrm{~ns}$, as used later in the experiment; however, note that the absolute SNR for the long PRBS is actually slightly lower due to the higher background component introducing noise into the signal. As detailed in the Supplementary material, the SNR grows with the pulse duration because (i) the acoustic wave has more time to grow and (ii) longer pulses require smaller detection bandwidth. The theoretical SNR shows a good agreement with the measurements for the pump pulse duration below $90 \mathrm{~ns}$ (Figure 5a), where the system's noise is given by the thermal noise of the detector. After $90 \mathrm{~ns}$, the SNR reaches an asymptotic value; for the short PRBS this response is caused by the overlap between responses of two consecutive peaks, whereas for the long PRBS this response is caused by distortions originating from the increased background signal.

Figure $5 \mathrm{~b}$ shows the measurement time as a function of the pump pulse duration. The amplitudes in both curves were normalized to the case of a pulse duration of $70 \mathrm{~ns}$ for a better comparison with our final experiment. However, due to the larger number of correlation peaks in the time-domain traces obtained with a short PRBS, the absolute measurement time with the short PRBS is actually 32 times shorter than the one for long PRBS. Figure $5 \mathrm{~b}$ shows that the minimum measurement time is obtained with pulses ranging between 60 and $90 \mathrm{~ns}$, which also corresponds to the range showing the maximum SNR in Figure 5a.

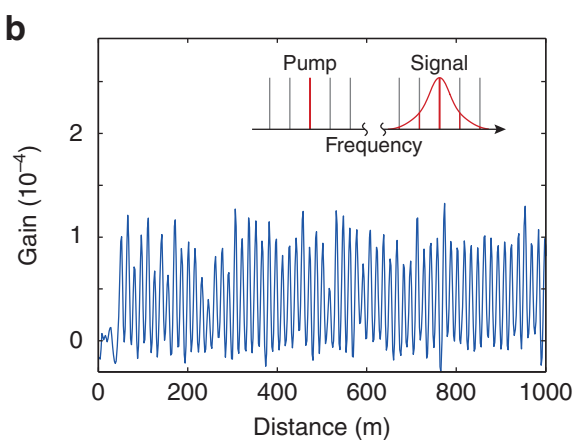

Figure 4 Measured Brillouin gain trace along the first kilometer of the sensing fiber, for (a) PRBS of $2^{15}-1$ bits and (b) PRBS of $2^{10}-1$ bits. Insets show schematics of the multiple Brillouin cross-interactions between pump and probe spectral components. 


\section{Measurement along a 17.5-km-long fiber}

Based on the previous pulse optimization, a pulse width of $70 \mathrm{~ns}$ and the short PRBS of $2^{10}-1$ bits (1023 bits) have been selected for our experiment to maximize the number of correlation peaks in the temporal traces and minimize the measurement time. Moreover, pulses of $70 \mathrm{~ns}$ ensure the absence of temporal overlapping between responses of consecutive correlation peaks. At the same time, the SNR achieved for this pulse duration corresponds to $\sim 90 \%$ of the maximum SNR (reached at $90 \mathrm{~ns}$ ), representing a negligible penalty (less than $0.5 \mathrm{~dB}$ ) with respect to the optimal SNR level.

A PRBS duration of 1023 bits (with bits of $140 \mathrm{ps}$ ) gives a correlation peak separation of $14.3 \mathrm{~m}$, allowing for a simultaneous measurement of $\sim 1200$ points along the 17.5 -km-long fiber in a single temporal trace. The Brillouin gain has been measured using 512 averaged temporal traces, whereas the Brillouin frequency has been retrieved by fitting the spectrum at each position with a quadratic curve $^{31}$. The results of this fitting are shown in Figure 6a. To estimate the uncertainty on the measurement, the standard deviation of the retrieved Brillouin frequency has been evaluated along the whole fiber by repeating the measurements multiple times. Figure $6 \mathrm{~b}$ depicts the uncertainty of the estimated Brillouin frequency as a function of distance, showing an expected exponential growth along the fiber, which reaches the average value of $1.8 \mathrm{MHz}$ at the farthest fiber end.

\section{Validation of high spatial resolution}

Our previous work $\mathrm{k}^{27}$ showed that a traditional hotspot technique is not suitable for the validation of very-high spatial resolutions due to the heat propagation along the fiber. For this demonstration, a sharp transition in the Brillouin frequency would be more appropriate.
In this case, the high spatial resolution of the implemented system is verified by measuring the Brillouin gain spectrum inside the FC-APC connectors at both ends of the sensing fiber (marked with red dots in Figure 3). Note that to mount the fiber connectors (such as standard FC-PC or FC-APC connectors), the optical fiber is glued inside an 8 -mm ceramic ferrule, which applies a strain to the fiber, resulting in a sharp shift of the Brillouin frequency.

The first measurement is performed for connector 1, linking the farthest end of the sensing fiber to the attached isolator. At this point, the system shows the lowest SNR, resulting in the best condition to verify the high spatial resolution of the system. Figure 7a shows the top view of the measured Brillouin gain spectrum and the retrieved Brillouin frequency (white circles) at each fiber position. It can be seen that the 16-mm-long section under strain from the two mated connectors is clearly resolved with very sharp transitions, demonstrating that the system is capable of resolving even the shorter segment with step Brillouin frequency changes (representing more than 1000000 points along the 17.5 -km-long fiber). Figure 7b shows the gain response of the sensor at the average peak gain frequency (outside connectors). The drop of the Brillouin gain, as a consequence of the strain inside the connectors, is clearly observed. In addition, the sensor response for a spatial resolution of $8.3 \mathrm{~mm}$ was theoretically estimated based on the model presented in Section S2 of the Supplementary material. This response is also illustrated in the figure (red dashed line), showing a good agreement with the experimental trace and validating the spatial resolution of the system.

To provide a second comparison, the measurement is repeated for connector 2 linking the circulator to the beginning of the sensing fiber. At this position, the SNR of the measurements shows the maximum
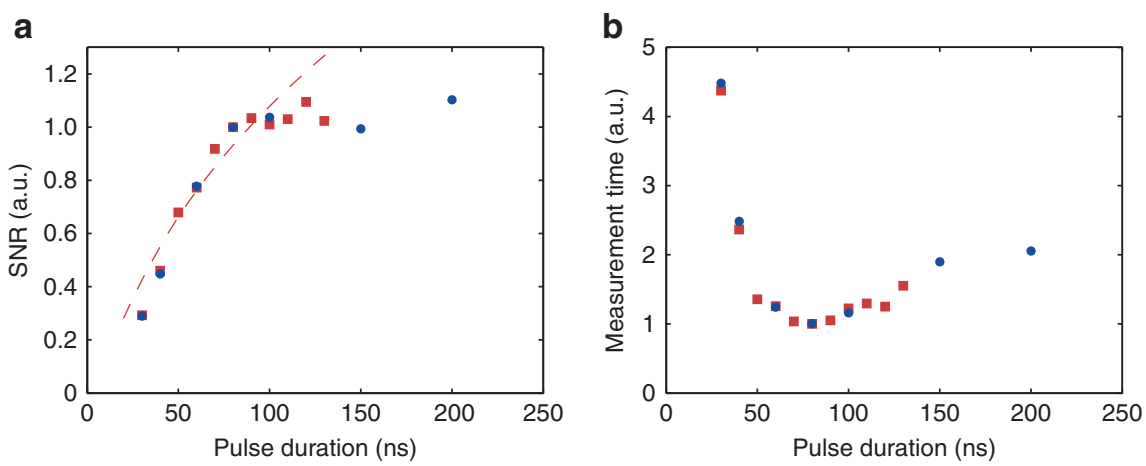

Figure 5 (a) Measured SNR versus pump pulse duration for short PRBS (red squares) and long PRBS (blue circles), and theoretically predicted SNR using Equation (S15) of the Supplementary material (dashed red line). (b) Relative measurement time versus pump pulse duration for short PRBS (red squares) and long PRBS (blue circles). Curves in both figures are normalized to the case of the 70-ns pulse.
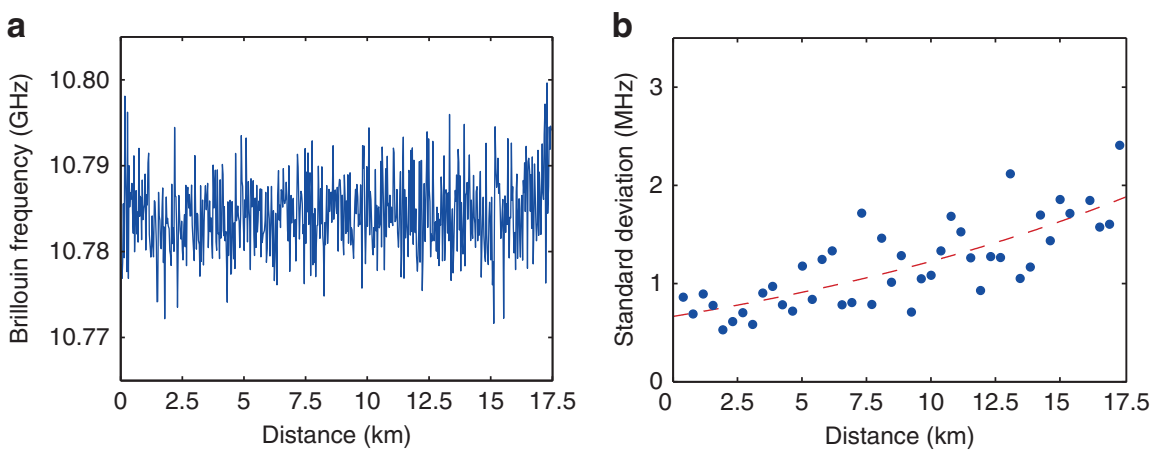

Figure 6 (a) Measured Brillouin frequency distribution along a 17.5-km-long fiber with 8.3-mm spatial resolution, and (b) frequency uncertainty versus distance (the dashed red line represents an exponential fitting of the measured points). 
a

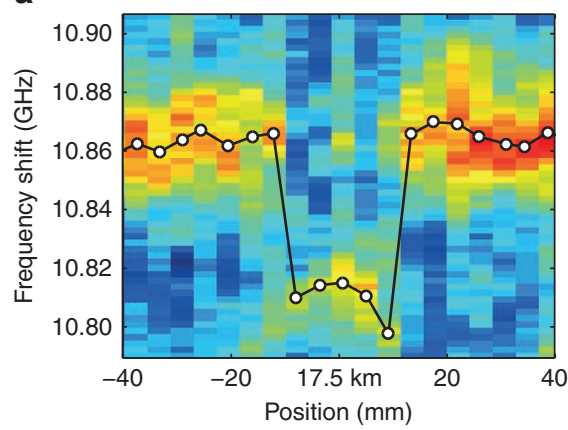

C

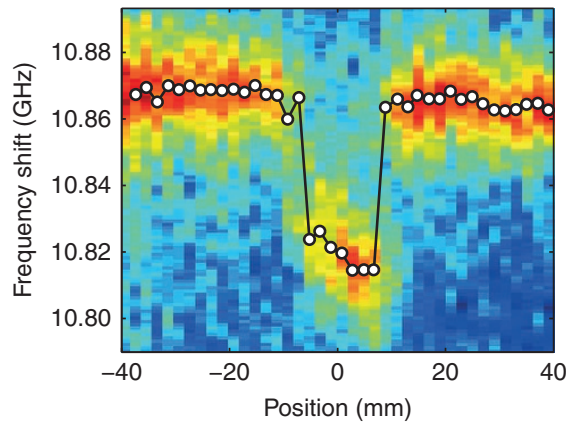

b

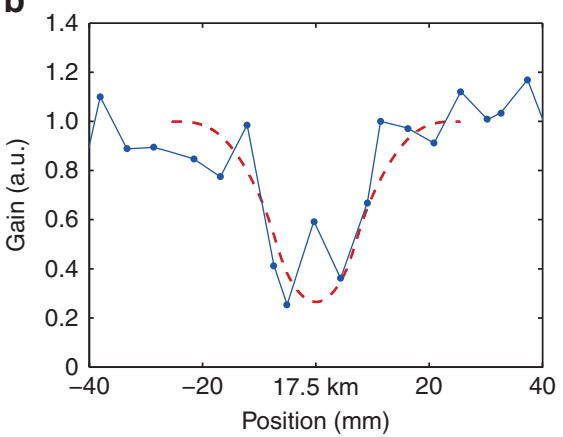

d

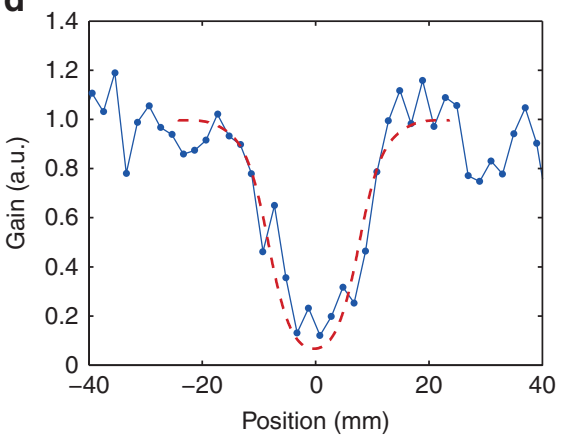

Figure 7 (a) Brillouin gain spectrum and extracted Brillouin frequency (white dots) inside the connector 1 at the farthest end of the 17.5-km-long fiber using 8.3-mm spatial resolution. (b) Gain for the average Brillouin frequency of the fiber: measured (blue line) and theoretically predicted (dashed red line). $(\mathbf{c}, \mathbf{d})$ Same information, but for the measurement of connector 2 at the beginning of the fiber (for illustrative purposes and to validate the model describing the spatial resolution; in this case, the spatial resolution has been improved down to $4.5 \mathrm{~mm}$ ).

value, so that it was possible to perform the measurement with the shortest bit duration ( $80 \mathrm{ps}$ ) provided by our PRBS generator. Using a sampling interval of $2 \mathrm{~mm}$, the attained spatial resolution is refined to $4.5 \mathrm{~mm}$. Figure $7 \mathrm{c}$ shows the measured Brillouin spectrum and the retrieved Brillouin frequency shift as a function of the fiber position, and Figure $7 \mathrm{~d}$ compares the experimental and theoretically predicted gain response versus fiber location at the average peak gain frequency. The good agreement between theory and experiment in both cases validates the model and the approach presented in Section S2 of the Supplementary material describing the spatial resolution of a phasecorrelated Brillouin sensor, which confirms that the spatial resolution obtained with a sampling interval of $4.7 \mathrm{~mm}$ is equal to $8.3 \mathrm{~mm}$, as described in Figure 2, corresponding to 2100000 points being measured along the whole sensing fiber.

\section{CONCLUSIONS}

In this study, we demonstrated a distributed fiber sensor capable of resolving 2100000 independent points along a single $17.5-\mathrm{km}$-long fiber. To the best of our knowledge, this is the highest number ever achieved by any distributed sensor. This remarkable performance was enabled by a detailed theoretical analysis of the system's limitations and an optimization process that provides the best possible SNR. The theoretical analysis performed in the current study can be a very useful tool to design a distributed fiber sensor based on the phase-correlation technique for medium-to-long range and sub-meter spatial resolution.

In its present state, a fully optimized measurement of 1000000 points in the fiber (spatial resolution of $17.5 \mathrm{~mm}$ ) covering 100 scanned frequencies (e.g., $100 \mathrm{MHz}$ span with a step of $1 \mathrm{MHz}$ ) requires $1.5 \mathrm{~h}$, assuming zero latency of the acquisition system. This performance is more than 20 times faster than the previously demonstrated result ${ }^{27}$, due to the changes in the experimental setup and the optimization of the bit duration described in Section S4 of the Supplementary material. The measurement time is determined by the inherent limitations of correlation-domain methods and is given by the large number of correlation peak positions that have to be scanned. However, there are multiple approaches for accelerating the measurement process. First, the measurement time scales cubically with the number of points, e.g., a measurement with $5-\mathrm{cm}$ resolution (corresponding to more than 300000 points) would take only 4 min, which opens an interesting opportunity for a new approach in distributed sensing in which one can start with an initial coarse scan with a spatial resolution of several centimeters and then proceed with a more detailed scan of detected regions of interest, which may not require a full scan of the whole fiber. Thus, all the points of interest can be scanned relatively quickly. Alternatively, one may loosen the requirement for the measurement uncertainty so that a reduced number of averages would be required to allow for faster measurements. Once again, this measurement can be followed by a more precise measurement of the detected points of interest.

The obtained results show that a deep understanding of the noise sources can lead to a significant improvement in the sensor performance. As shown in Ref. 29, amplitude coding techniques used in pure time-domain systems can be readily applied to achieve an additional improvement in SNR, thus allowing for much faster measurements. For example, an amplitude code of 1000 bits might improve the SNR of the measurements by a factor of 10 , allowing for a 100 -fold decrease of the number of averages. In this way, the measurement time of $1.5 \mathrm{~h}$ would be reduced down to $1 \mathrm{~min}$.

\section{ACKNOWLEDGEMENTS}

This work was supported by the Swiss National Science Foundation through the project 200021-134546 and the Swiss State Secretariat for Education, Research and Innovation (SERI) through the project COST C10.0093. 
1 Horiguchi T, Tateda M. BOTDA-nondestructive measurement of single-mode optical fiber attenuation characteristics using Brillouin interaction: theory. J Lightwave Technol 1989; 7: 1170-1176.

2 Rodriguez-Barrios F, Martin-Lopez S, Carrasco-Sanz A, Corredera P, Ania-Castanon J et al. Distributed Brillouin fiber sensor assisted by first-order Raman amplification. J Lightwave Technol 2010; 28: 2162-2172.

3 Martin-Lopez S, Alcon-Camas M, Rodriguez F, Corredera P, Ania-Castañon JD et al. Brillouin optical time-domain analysis assisted by second-order Raman amplification. Opt Express 2010; 18: 18769-18778.

4 Soto MA, Bolognini G, Di Pasquale F. Optimization of long-range BOTDA sensors with high resolution using first-order bi-directional Raman amplification. Opt Express 2011; 19: 4444-4457.

5 Angulo-Vinuesa X, Martin-Lopez S, Corredera P, Gonzalez-Herraez M. Raman-assisted Brillouin optical time-domain analysis with sub-meter resolution over $100 \mathrm{~km}$. Opt Express 2012; 20: 12147-12154.

6 Soto MA, Taki M, Bolognini G, Di Pasquale F. Simplex-coded BOTDA sensor over 120$\mathrm{km}$ SMF with 1-m spatial resolution assisted by optimized bidirectional Raman amplification. IEEE Photon Technol Lett 2012; 24: 1823-1826.

7 Soto MA, Bolognini G, Di Pasquale F, Thévenaz L. Simplex-coded BOTDA fiber sensor with $1 \mathrm{~m}$ spatial resolution over a $50 \mathrm{~km}$ range. Opt Lett 2010; 35: 259-261.

8 Soto MA, Bolognini G, Di Pasquale F. Analysis of optical pulse coding in spontaneous Brillouin-based distributed temperature sensors. Opt Express 2008; 16: 19097-19111.

9 Soto MA, Bolognini G, Di Pasquale F. Analysis of pulse modulation format in coded BOTDA sensors. Opt Express 2010; 18: 14878-14892.

10 Liang $\mathrm{H}$, Li WH, Linze N, Chen L, Bao XY. High-resolution DPP-BOTDA over $50 \mathrm{~km}$ LEAF using return-to-zero coded pulses. Opt Lett 2010; 35: 1503-1505.

11 Soto MA, Bolognini G, Di Pasquale F. Long-range simplex-coded BOTDA sensor over $120 \mathrm{~km}$ distance employing optical preamplification. Opt Lett 2011; 36: 232-234.

12 Jia XH, Rao YJ, Wang ZN, Zhang WL, Yuan CX et al. Distributed Raman amplification using ultra-long fiber laser with a ring cavity: characteristics and sensing application. Opt Express 2013; 21: 21208-21217.

13 Soto MA, Angulo-Vinuesa X, Martin-Lopez S, Chin SH, Ania-Castanon J et al. Extending the real remoteness of long-range Brillouin optical time-domain fiber analyzers. J Lightwave Technol 2014; 32: 152-162.

14 Fellay $A$, Thévenaz $L$, Facchini $M$, Niklès $M$, Robert $P$. Distributed sensing using stimulated Brillouin scattering: towards ultimate resolution. Proceedings of the 12th International Conference on Optical Fiber Sensors. OSA: Washington DC, USA, 1997, ppOWD31-OWD34.

15 Hotate K, Hasegawa T. Measurement of Brillouin gain spectrum distribution along an optical fiber using a correlation-based technique-proposal, experiment and simulation. IEICE Trans Electr 2000; 83: 405-412.

16 Mizuno Y, Zou WW, He ZY, Hotate K. Proposal of Brillouin optical correlation-domain reflectometry (BOCDR). Opt Express 2008; 16: 12148-12153.

17 Zadok A, Antman Y, Primerov N, Denisov A, Sancho J et al. Random-access distributed fiber sensing. Laser Photon Rev 2012; 6: L1-L5.

18 Soto MA, Chin S, Thévenaz L, Double-pulse Brillouin distributed optical fiber sensors: analytical model and experimental validation. Proc SPIE 2012; 8421: 842124.
19 Brown AW, Colpitts BG, Brown K. Distributed sensor based on dark-pulse Brillouin scattering. IEEE Photon Technol Lett 2005; 17: 1501-1503.

20 Song KY, Zou WW, He ZY, Hotate K. All-optical dynamic grating generation based on Brillouin scattering in polarization-maintaining fiber. Opt Lett 2008; 33: 926-928.

$21 \mathrm{Li} \mathrm{WH}$, Bao XY, Li Y, Chen L. Differential pulse-width pair BOTDA for high spatial resolution sensing. Opt Express 2008; 16: 21616-21625.

22 Foaleng S, Tur M, Beugnot JC, Thevenaz L. High spatial and spectral resolution longrange sensing using Brillouin echoes. J Lightwave Technol 2010; 28: 2993-3003.

23 Dong YK, Zhang HY, Chen L, Bao XY. $2 \mathrm{~cm}$ spatial-resolution and $2 \mathrm{~km}$ range Brillouin optical fiber sensor using a transient differential pulse pair. Appl Opt 2012; 51: 1229-1235.

24 Soto MA, Thévenaz L, Towards 1'000'000 resolved points in a distributed optical fibre sensor. Proc SPIE 2014; 9157: $9157 \mathrm{C3}$.

25 Soto MA, Taki M, Bolognini G, Di Pasquale F. Optimization of a DPP-BOTDA sensor with $25 \mathrm{~cm}$ spatial resolution over $60 \mathrm{~km}$ standard single-mode fiber using Simplex codes and optical pre-amplification. Opt Express 2012; 20: 6860-6869.

26 Denisov A, Soto MA, Thévenaz LTime gated phase-correlation distributed Brillouin fibre sensor. Proc SPIE 2013; 8794: 87943I.

27 Denisov A, Soto MA, Thévenaz L. 1'000'000 resolved points along a Brillouin distributed fibre sensor. Proc SPIE 2014; 9157: 9157D2.

28 Elooz D, Antman Y, Levanon N, Zadok A. High-resolution long-reach distributed Brillouin sensing based on combined time-domain and correlation-domain analysis. Opt Express 2014; 22: 6453-6463.

29 London Y, Antman Y, Cohen R, Kimelfeld N, Levanon N et al. High-resolution longrange distributed Brillouin analysis using dual-layer phase and amplitude coding. Opt Express 2014; 22: 27144-27158.

30 Antman Y, Primerov N, Sancho J, Thevenaz L, Zadok A. Localized and stationary dynamic gratings via stimulated Brillouin scattering with phase modulated pumps. Opt Express 2012; 20: 7807-7821.

31 Soto MA, Thévenaz L. Modeling and evaluating the performance of Brillouin distributed optical fiber sensors. Opt Express 2013; 21: 31347-31366.

32 Foaleng SM, Thévenaz L, Impact of Raman scattering and modulation instability on the performances of Brillouin sensors. Proc SPIE 2011; 7753: 77539V.

33 Alem M, Soto MA, Thévenaz L. Analytical model and experimental verification of the critical power for modulation instability in optical fibers. Opt Express 2015; 23: 29514-29532.

34 Zhou JH, Chen JP, Jaouen Y, Yi LL, Petit $\mathrm{H}$ et al. A new frequency model for pump-tosignal RIN transfer in Brillouin fiber amplifiers. IEEE Photon Technol Lett 2007; 19: 978-980.

35 David A, Horowitz M. Low-frequency transmitted intensity noise induced by stimulated Brillouin scattering in optical fibers. Opt Express 2011; 19: 11792-11803.

(c) (i) (9) This work is licensed under a Creative Commons Attributioncc. other third party material in this article are included in the article's Creative Commons license, unless indicated otherwise in the credit line; if the material is not included under the Creative Commons license, users will need to obtain permission from the license holder to reproduce the material. To view a copy of this license, visit http:// creativecommons.org/licenses/by-nc-sa/4.0/

Supplementary Information for this article can be found on the Light: Science \& Applications' website (http://www.nature.com/lsa). 